\title{
Fast In-place Integer Radix Sorting
}

\author{
Fouad El-Aker \\ Computer Science Department, University of Petra, \\ P.O. Box 940650, Amman 11194, Jordan \\ elaker_fouad@maktoob.com \\ elaker_fouad@yahoo.ca
}

\begin{abstract}
This paper presents two very efficient sorting algorithms. MSL is an $\mathrm{O}\left(N^{*} B\right)$ in-place radix sorting algorithm, where $N$ is the input size and $B$ is the keys length in bits. This paper presents an implementation of MSL that is sublinear in practice, for uniform data, on Pentium 4 machines. We also present an $\mathrm{O}\left(N^{*} \log N\right)$ hybrid quicksort that has a non-quadratic worst case.
\end{abstract}

\section{Introduction}

Right to left LSD and left to right MSD are $\mathrm{O}\left(N^{*} B\right)$ radix sorting algorithms. $N$ is the input size and $B$ is the length of keys in bits. LSD and MSD use an extra space of size $N$. ALR [4] and MSL [1] process bits left to right, however unlike MSD, ALR and MSL are in-place and cache friendly. MSD, ALR and MSL execute recursively, for every partition. LSD body code is executed only $B / D$ times, where $B$ is the length of keys in bits and $D$ is the used digit size. This makes the design of algorithms faster than LSD quite difficult. LSD is faster than MSD in [6]. We present a sub-linear run time MSL implementation suitable for sorting 31 bits and 63 bits integers in Java in this paper. MSL implementation in Section 2 uses small digit sizes increasing data cache friendliness. MSL loops were implemented reducing the number of instructions, and therefore increasing instruction cache friendliness. In addition, section 3 presents a non-quadratic implementation of quicksort, called switch sort. Hybridizing switch sort and MSL does not improve over MSL. Section 4 presents the test results. Section 5 gives the conclusions and future work.

\section{MSL and Smaller Digit Sizes}

MSL and ALR use a permutation loop in order to avoid reserving an extra array of size $N$, which is performed in MSD. The main steps of MSL are presented in [1]. MSL permutation loop, shuffles keys into their target groups. In the circular list of keys, $\mathrm{K}=\left\langle\mathrm{K}_{1}, \mathrm{~K}_{2}, . ., \mathrm{K}_{\mathrm{L}}>\right.$, assume the Target Address $\left(\mathrm{K}_{\mathrm{J}}\right)=$ Array Location $\left(\mathrm{K}_{\mathrm{J}+1}\right)$, where $\mathrm{J}$ is not equal to $\mathrm{L}$, and Target Address $\left(\mathrm{K}_{\mathrm{L}}\right)=$ Array Location $\left(\mathrm{K}_{1}\right)$. Digit extraction and group end address lookup are used in computing a key's target address. MSL permutation loop moves keys in a circular list $\mathrm{K}$ to their target addresses. $\mathrm{K}_{1}$ is named the initial key in $\mathrm{K}$, and is computed prior to the permutation loop. 
Many permutation loops are required in order to shuffle all keys in a group to their target addresses. ALR searches for $\mathrm{K}_{1}$ sequentially, and preserves the property that all keys to the left of the current $\mathrm{K}_{1}$ key are in their correct target addresses. MSL searches groups' information sequentially for the left most group, $\mathrm{G}_{\text {Left }}$, which has at least one key possibly not in its target address. MSL uses the top key in $\mathrm{G}_{\text {Left }}$, as $\mathrm{K}_{1}$.

In [5], sections 4 and 5, digit size 6 was determined as appropriate for radix sorting algorithms. This is because of data cache friendliness. The importance of cache friendliness in radix sorting algorithms is emphasized in [5] and [4]. MSL also cuts to insertion sort for group sizes 20 or less, same as [4].

\section{Switch Sort}

Hybridized quicksort [3] implementation in this paper selects one from many pivot computations and is described in this section. Assume that we are interleaving the execution of a constant number, $K$, of divide and conquer algorithms whose worst cases are $f_{l}, f_{2}, . . f_{K}$. The list of algorithms is denoted $A L=\left(A_{l}, A_{2}, . . A_{K}\right)$. When $A_{J}$ in $A L$ performance is degenerate, we interrupt $A_{J}$ and switch execution to the next algorithm in the circular list $A L$. $A L$ worst case is equal to $K * f_{W}$ provided that the following conditions hold. (1) $f_{W}=\operatorname{Min}\left(f_{1}, f_{2}, . . f_{K}\right)$. (2) We can determine that the current call to $A_{J}$ is futile in constant time. (3) We can switch execution to the next algorithm in the circular list $A L$ without loosing the processing done so far. If each $A_{J}$ in $A L$ executes a futile call, execution returns to $A_{W}$ after circular calls to other algorithms in $A L$. A quicksort example is shown and described next.

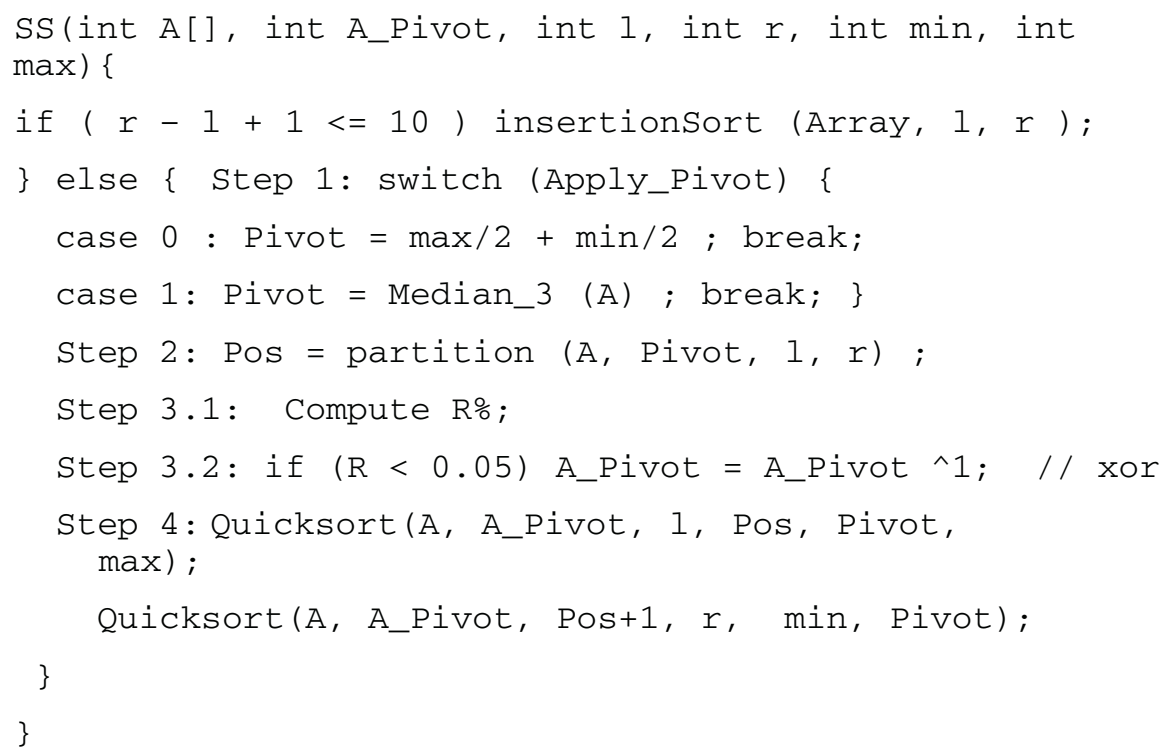


We measure balanced partitioning in quicksort to determine that the current call is futile. The partitioning ratio is defined as the size of the smaller partition divided by the size of the input group in quicksort. Let $\mathrm{P} \%$ be the minimum acceptable partitioning ration, over all the algorithms in $A L$, equals $5 \%$ in Step 3.2. $\mathrm{R} \%$ is the partitioning ration for the current quicksort call. When $\mathrm{R} \%<\mathrm{P} \%$, Step 3.2, partitioning is named degenerate or a failure. $A L$ code above has only quicksort implementations, and a switch statement is used to decide which pivot computation to use, see Step 1 above. We call the algorithm switch sort (SS). Step 3.2 selects an alternative pivot computation for recursive calls. Max-Min average pivot computation in the first line in Step 1 is an adaptive implementation of taking the middle value of the input range in radix exchange [7]. Median of three quicksort passes down the actual lower partition max and the actual upper partition min. Radix exchange always divides the input range by half on recursive calls, independent of data. $A L$ worst case is $O(2 * N \lg N)$, where the worst case of radix exchange is $O(2 * N \lg N)$.

\section{Experimental Results}

In Table 1, MSL run time is non-significantly sub-linear in experiments. The test data is uniform. The machine used for the displayed results is $3 \mathrm{GHz}$ Pentium 4, $1 \mathrm{~GB}$ RAM, 1MB level 2 cache, and $16 \mathrm{~KB}$ level 1 cache, with $400 \mathrm{MHz}$ RAM speed. MSL sub-linear run time was confirmed on other Pentium 4 machines. In Table 1, add the sizes at columns headings to the sizes at each row to get the array size at a cell. Row $30 \mathrm{M}+(30$ millions+) and column $+5 \mathrm{M}$ refer to the cell for the array size 35 millions.

In Table 1, MSL running time for array size 35 millions is 4000 milliseconds, and for array size 70 millions is 7875 milliseconds, 31 bits integers. In Table 1, the running time for array size 25 millions, is 4032 milliseconds, and for array size 50 millions, is 7735 milliseconds, for 63 bits integers.

Cutting to insertion sort is an important factor in MSL. On the other hand, we could not improve the running time of MSL by hybridizing MSL with switch sort. MSL and switch sort are compared against other algorithms in Table 2.

Table 1. MSL running times in milliseconds. Sizes are multiple of $M=10^{6}$

\begin{tabular}{|l|c|c|c|c|c|c|c|}
\hline 31Bits & $+1 \mathrm{M}$ & $+2 \mathrm{M}$ & $+4 \mathrm{M}$ & $+5 \mathrm{M}$ & $+6 \mathrm{M}$ & $+8 \mathrm{M}$ & $+10 \mathrm{M}$ \\
\hline $\mathrm{N}=0+$ & 93 & 188 & 437 & 657 & 890 & 1234 & 1469 \\
\hline $\mathrm{N}=10 \mathrm{M}+$ & 1563 & 1671 & 1875 & 1984 & 2094 & 2266 & 2500 \\
\hline $\mathrm{N}=20 \mathrm{M}+$ & 2578 & 2678 & 2891 & 2984 & 3078 & 3281 & 3484 \\
\hline $\mathrm{N}=30 \mathrm{M}+$ & 3594 & 3703 & 3906 & 4000 & 4125 & 4344 & 4547 \\
\hline $\mathrm{N}=40 \mathrm{M}+$ & 4671 & 4766 & 5000 & 5079 & 5218 & 5390 & 5625 \\
\hline $\mathrm{N}=50 \mathrm{M}+$ & 5782 & 5875 & 6062 & 6172 & 6313 & 6532 & 6734 \\
\hline $\mathrm{N}=60 \mathrm{M}+$ & 6890 & 6953 & 7172 & 7313 & 7453 & 7656 & 7875 \\
\hline $\mathbf{6 3 B i t s}$ & $+1 \mathrm{M}$ & $+2 \mathrm{M}$ & $+4 \mathrm{M}$ & $+5 \mathrm{M}$ & $+6 \mathrm{M}$ & $+8 \mathrm{M}$ & $+10 \mathrm{M}$ \\
\hline $\mathrm{N}=0+$ & 141 & 250 & 594 & 843 & 1125 & 1531 & 1859 \\
\hline $\mathrm{N}=10 \mathrm{M}+$ & 2016 & 2157 & 2485 & 2594 & 2765 & 3016 & 3281 \\
\hline $\mathrm{N}=20 \mathrm{M}+$ & 3469 & 3578 & 3859 & 4032 & 4141 & 4438 & 4735 \\
\hline $\mathrm{N}=30 \mathrm{M}+$ & 4860 & 5016 & 5328 & 5469 & 5625 & 5891 & 6203 \\
\hline $\mathrm{N}=40 \mathrm{M}+$ & 6375 & 6500 & 6797 & 6953 & 7125 & 7406 & 7735 \\
\hline
\end{tabular}


LSD, digit size 8 (LSD8) is faster than LSD with digit size 16, LSD16, and other digit sizes, on the test machine. LSD processes the total keys bits. MSL processes only the distinguishing prefixes, but is recursive (section 1). In Table 2, MSL has half the run time of LSD8 for 63 bits data. In addition, MSL is better than LSD8 for larger 31 bits arrays. See size 16 and 32 millions as well as MSL sub-linear run time in Table 1. Switch sort (SS), is faster than LSD16, 63 bits longs data. Switch sort is also faster than the two algorithms, which Switch sort alternates, quicksort and Max-Min Average (MMA). Java built in tuned quicksort (JS), which is a tuned implementation of [2], is used in Table 2, instead of our own slower median of three quicksort.

Table 2. MSL running times in milliseconds. Sizes are multiple of $M=10^{6}$

\begin{tabular}{|l|l|l|l|l|l|l|l|}
\hline 31Bits & $1 / 2 \mathrm{M}$ & $1 \mathrm{M}$ & $2 \mathrm{M}$ & $4 \mathrm{M}$ & $8 \mathrm{M}$ & $16 \mathrm{M}$ & $32 \mathrm{M}$ \\
\hline MSL & 47 & 93 & 188 & 437 & 1234 & 2094 & 3703 \\
\hline LSD8 & 47 & 109 & 234 & 454 & 938 & 1875 & 3859 \\
\hline LSD16 & 94 & 234 & 500 & 1031 & 2047 & 4250 & 8656 \\
\hline JS & 109 & 234 & 516 & 1062 & 2219 & 4640 & 9672 \\
\hline SS & 109 & 250 & 500 & 1031 & 2141 & 4500 & 9546 \\
\hline MMA & 109 & 234 & 500 & 1031 & 2172 & 4516 & 9438 \\
\hline 63Bits & $1 / 2 \mathrm{M}$ & $1 \mathrm{M}$ & $2 \mathrm{M}$ & $4 \mathrm{M}$ & $8 \mathrm{M}$ & $16 \mathrm{M}$ & $32 \mathrm{M}$ \\
\hline MSL & 62 & 141 & 250 & 594 & 1531 & 2765 & 5016 \\
\hline LSD8 & 172 & 344 & 672 & 1328 & 2719 & 5563 & 10953 \\
\hline LSD16 & 250 & 516 & 1015 & 2031 & 4563 & 8609 & 18891 \\
\hline JS & 156 & 329 & 672 & 1422 & 2969 & 6203 & 12922 \\
\hline SS & 140 & 313 & 640 & 1344 & 2781 & 5860 & 12203 \\
\hline MMA & 141 & 312 & 641 & 1360 & 2829 & 5906 & 12359 \\
\hline
\end{tabular}

\section{Conclusion and Future Work}

MSL is a sub-linear in-place radix-sorting algorithm, for uniform data. Switch sort is a non-quadratic implementation of quicksort. Future work includes low run time algorithms and models for sorting as well as for other problems.

\section{References}

1. Al-Badarneh Amer, El-Aker Fouad: Efficient In-Place Radix Sorting, Informatica, 15 (3), 2004, pp. 295-302.

2. J. L. Bentley, and M. D. Mcllroy: Engineering a Sort Function, Software-Practice and Experience, 23 (1), 1993, pp. 1249-1265.

3. F. El-Aker, and A. Al-Badarneh: MSL: An Efficient Adaptive In-place Radix Sorting Algorithm, ICCS, Part II, 2004, pp. 606-609.

4. Maus, A.: ARL: A Faster In-place, Cache Friendly Sorting Algorithm, Norsk Informatikkonferranse, NIK'2002, 2002, pp. 85-95.

5. N. Rahman and R. Raman: Adapting radix sort to the memory hierarchy, Proc. $2^{\text {nd }}$ Workshop on Algorithm Engineering and Experiments, ALENEX, 2000.

6. Sedgewick, R.: Algorithms in Java, Parts 1-4, $3^{\text {rd }}$ Ed., Addison-Wesley, 2003. 